\title{
Functional Consequences of Pravastatin Isomerization on OATP1B1-Mediated Transport ${ }^{\mathbb{S}}$
}

\author{
Jonathan B. Wagner, Melissa Ruggiero, J. Steven Leeder, and \Bruno Hagenbuch
}

Ward Family Heart Center (J.B.W.) and Division of Clinical Pharmacology, Toxicology and Therapeutic Innovation (J.B.W., J.S.L.), Children's Mercy, Kansas City, Missouri; Department of Pediatrics, University of Missouri-Kansas City School of Medicine, Kansas City, Missouri (J.B.W., J.S.L.); and Department of Pharmacology, Toxicology, and Therapeutics, The University of Kansas Medical Center, Kansas City, Kansas (M.R., B.H.)

Received May 19, 2020; accepted August 17, 2020

\section{ABSTRACT}

Pravastatin acid (PVA) can be isomerized to its inactive metabolite

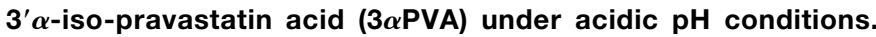
Previous studies reported interindividual differences in circulating concentrations of PVA and $3 \alpha$ PVA. This study investigated the functional consequences of PVA isomerization on OATP1B1mediated transport. We characterized 3 $\alpha$ PVA inhibition of OATP1B1mediated PVA uptake into human embryonic kidney 293 cells expressing the four different OATP1B1 proteins $\left({ }^{*} 1 \mathrm{a},{ }^{*} \mathbf{1 b},{ }^{*} 5\right.$, and *15). 3 $\alpha$ PVA inhibited OATP1B1-mediated PVA uptake in all four OATP1B1 gene products but with lower $I C_{50} / K_{i}$ values for OATP1B1*5 and ${ }^{*} 15$ than for the reference proteins $\left({ }^{*} 1 a\right.$ and $\left.{ }^{*} 1 b\right)$. PVA and $3 \alpha$ PVA were transported by all four OATP1B1 proteins. Kinetic experiments revealed that maximal transport rates $\left(\mathrm{V}_{\max }\right.$ values) for OATP1B1 variants $* 5$ and ${ }^{*} 15$ were lower than for ${ }^{*} 1 a$ and $* 1 b$ for both substrates. Apparent affinities for $3 \alpha$ PVA transport were similar for all four variants. However, the apparent affinity of OATP1B1*5 for $3 \alpha$ PVA was higher (lower $K_{m}$ value) than for PVA. These data confirm that PVA conversion to $3 \alpha$ PVA can have functional consequences on PVA uptake and impacts OATP1B1 variants more than the reference protein, thus highlighting another source variation that must be taken into consideration when optimizing the PVA dose-exposure relationship for patients.

\section{SIGNIFICANCE STATEMENT}

$3^{\prime} \alpha$-iso-pravastatin acid inhibits pravastatin uptake for all OATP1B1 protein types; however, the $\mathbf{I C}_{50}$ values were significantly lower in OATP1B1*5 and ${ }^{*} 15$ transfected cells. This suggests that a lower concentration of $3^{\prime} \alpha$-iso-pravastatin is needed to disrupt OATP1B1-mediated pravastatin uptake, secondary to decreased cell surface expression of functional OATP1B1 in variant-expressing cells. These data will refine previous pharmacokinetic models that are utilized to characterize pravastatin interindividual variability with an ultimate goal of maximizing efficacy at the lowest possible risk for toxicity.

\section{Introduction}

Pravastatin, administered in its active hydroxyl acid form [pravastatin acid (PVA)] (Pan, 1991; Serajuddin et al., 1991; Shitara and Sugiyama, 2006), is subject to presystemic conversion to an inactive isomer, $3^{\prime} \alpha$-iso-pravastatin acid ( $3 \alpha \mathrm{PVA}$ ), in the acidic environment of the stomach (Everett et al., 1991; Triscari et al., 1995). When delivered directly to the stomach, via nasogastric tube, there was a 5-fold increase in $3 \alpha \mathrm{PVA}$ production relative to direct administration to the more basic duodenum (Triscari et al., 1995). Interindividual differences in the relative amounts of circulating PVA and $3 \alpha$ PVA have been observed in adults and children (Ito, 1998; Maeda et al., 2006; Wagner et al., 2019).

This work was supported by a Clinical and Translational Science Awards (CTSA) grant from National Center for Advancing Translational Sciences (NCATS) awarded to the University of Kansas for Frontiers: University of Kansas Clinical and Translational Science Institute [Grants UL1TR002366, KL2TR002367] and by National Institutes of Health [Grants RR021940, GM077336]. The contents are solely the responsibility of the authors and do not necessarily represent the official views of the National Institutes of Health or National Center for Advancing Translational Sciences.

https://doi.org/10.1124/dmd.120.000122.

S This article has supplemental material available at dmd.aspetjournals.org.
The clinical consequences of increased presystemic conversion to inactive $3 \alpha \mathrm{PVA}$ are diminished PVA plasma concentrations and reduced delivery of active drug to the hepatocyte, subsequently resulting in diminished intrahepatic PVA concentrations. Ultimately, this leads to a reduced pharmacodynamic effect (e.g., low-density lipoprotein cholesterol reduction) (Ito, 1998).

PVA remains the primary drug to prevent coronary allograft vasculopathy (CAV), the leading cause of chronic rejection occurring in approximately one out of every four to five pediatric cardiac transplant recipients (Mahle et al., 2005). This is in part because it does not undergo cytochrome P450-mediated metabolism (Everett et al., 1991), lowering the potential for drug-drug interaction with immunosuppressants. Additionally, PVA is initiated in children with familial hypercholesterolemia to attenuate atherosclerotic precursors of coronary artery disease (Luirink et al., 2019). Since it is the most hydrophilic statin, passive diffusion across the maturing tissues (e.g., brain, skeletal muscle) is minimal, and PVA has been proposed as one of the safer statins for the maturing child (Wagner and Abdel-Rahman, 2016). However, a large range of variability in cholesterol reduction in the children treated with pravastatin has been observed (Wagner and Abdel-Rahman, 2016).

After gastric transit and intestinal absorption, PVA undergoes hepatocellular uptake via the liver-specific hepatic drug transporter

ABBREVIATIONS: CAV, coronary allograft vasculopathy; HEK, human embryonic kidney; $3 \alpha$-PVA, $3^{\prime} \alpha$-iso-pravastatin acid; PVA, pravastatin acid; SNP, single nucleotide polymorphism; TBS, Tris-buffered saline. 
OATP1B1, encoded by the SLCO1B1 gene (Hsiang et al., 1999; Nakai et al., 2001). Two nonsynonymous single nucleotide polymorphisms (SNPs) in SLCO1B1 (c.388A $>$ G, c.521T $>$ C) are observed frequently among the general population (Pasanen et al., 2006). Collectively, these two SNPs comprise four different haplotypes: the reference sequence $* 1 a($ c.388A-c.521T) in addition to *1b (c.388G-c.521T), *5 (c.388Ac.521C), and $* 15$ (c.388G-c.521C). The c.521T $>$ C SNP (rs4149056), located in exon 5 of the $S L C O 1 B 1$ gene, results in a nonsynonymous amino acid change (Val174Ala). This change results in impaired transporter localization at the plasma membrane (Kameyama et al., 2005), leading to decreased uptake into human hepatocytes and increased pravastatin plasma concentrations, referred to as "systemic exposure" (Mwinyi et al., 2004; Niemi et al., 2004). As a result of increased systemic exposure, clinically significant adverse events, including statin-associated muscle symptoms, have been reported. They are more frequent in the presence of c.521C alleles (Link et al., 2008; Voora et al., 2009). Associations of this SNP with attenuated lipid lowering with statin therapy have previously been demonstrated (Tachibana-Iimori et al., 2004; Niemi et al., 2005; Li et al., 2015), although its impact on long-term pravastatin response remains equivocal (Igel et al., 2006; Martin et al., 2012; Dai et al., 2015).

Our previous investigation in children and adolescents confirmed a positive association between SLCO1B1 genotype and pravastatin exposure similar to observations in adults (Niemi et al., 2004; Wagner et al., 2019). Perhaps more significant was the observation of an approximate 10-fold range of PVA systemic exposure within both the reference (c.521TT) and variant (c.521TC) genotype groups (Wagner et al., 2019). One of the contributing factors to interindividual variability in PVA systemic exposure was the magnitude of presystemic conversion of PVA to $3 \alpha \mathrm{PVA}$ across the study cohort with the extent of isomerization being more impactful in those with c.521TC genotype (Wagner et al., 2019).

A previous report suggested that $3 \alpha \mathrm{PVA}$ is a substrate of OATP1B1 (Maeda et al., 2006). In fact, among our pediatric cohort, we observed an increase in $3 \alpha$ PVA systemic exposure in those participants with a SLCO1B1 c.521T $>$ C variation (Wagner et al., 2019) Additionally, among adult cohorts, trends toward higher $3 \alpha$ PVA systemic exposure with SLCO1B1 c.521T >C variation were observed (Maeda et al., 2006; Suwannakul et al., 2008). These in vivo data and limited in vitro data illustrating cellular uptake of $3 \alpha$ PVA suggest that $3 \alpha$ PVA is a substrate of OATP1B1.

Given the large degree of interindividual variability in PVA systemic exposure observed within genotype groups, especially the c.521TC patients, we wanted to test the hypothesis that inhibition of PVA uptake by $3 \alpha \mathrm{PVA}$ could be an additional covariate that alters the systemic and hepatic exposure of PVA in children and adults. In the present study, we confirmed that PVA and $3 \alpha \mathrm{PVA}$ are OATP1B1 substrates and that variant OATP1B1 transporters had reduced uptake relative to fully functional reference transporters.

\section{Materials and Methods}

Materials. Radiolabeled $\left[{ }^{3} \mathrm{H}\right]$-pravastatin sodium salt and $\left[{ }^{3} \mathrm{H}\right]-3^{\prime} \alpha$-isopravastatin sodium salt were purchased from American Radiolabeled Chemicals, Inc. (St. Louis, MO). Nonradioactive pravastatin sodium salt and $3^{\prime} \alpha$-iso-pravastatin sodium salt used were from Sigma Aldrich (St. Louis, MO) and Toronto Research Chemicals (Toronto, OR, Canada), respectively.

Plasmids and Cells Lines. Site-directed mutagenesis was performed utilizing a QuikChange Lighting Multi Site-Directed Mutagenesis Kit (Agilent Technologies, Santa Clara, CA) according to manufacturer's guidelines to generate SLCO1B1 genotypes of interest $(* 1 a, * 1 b, * 5, * 15)$. In short, point mutations were introduced into the pcDNA5/FRT expression vector containing the open reading frame of OATP1B $1 * 1 \mathrm{~b}$ using thermal cycling followed by digestion of the template DNA with DpnI (Gui and Hagenbuch, 2008). Oligonucleotides containing 388A $>\mathrm{G}$ and $521 \mathrm{~T}>\mathrm{C}$ were used to create OATP1B1*1a and OATP1B $1 * 15$ expression vectors, respectively. Then, c.521T $>\mathrm{C}$ was also introduced into the OATP1B1*1a vector to create the OATP1B $1 * 5$ expression vector. Mutagenic primers used for sitedirected mutagenesis were as follows: A388G , 5' -TCTAAAGAAACTAATATC AATTCATCAGAAAATTCAACA-3' (forward), and 5'-TGTTGAATTTTC TGATGAATTGATATTAGTTTCTTTAGA-3' (reverse); T521C, 5' -TCATAC ATGTGGATATATGCGTTCATGGGTAATATGCTT-3' (forward), 5'-AAG CATATTACCCATGAACGCATATATCCACATGTATGA-3' (reverse). The polymerase chain reaction site-directed mutagenesis was performed under the following conditions: 2-minute denaturation/activation at $95^{\circ} \mathrm{C}$ followed by 30 cycles with 20 -second denaturation at $95^{\circ} \mathrm{C}, 30$-second annealing at $55^{\circ} \mathrm{C}$, and 5 -minute elongation at $65^{\circ} \mathrm{C}$. The presence of the mutations was verified by sequencing all inserts in both directions. The resulting plasmids were used for transient transfection of human embryonic kidney (HEK) 293 cells.

Tissue Culture and Transporter Expression. HEK293/T17 cells (American Type Culture Collection, Manassas, VA) were grown at $37^{\circ} \mathrm{C}$ in a humidified $5 \% \mathrm{CO}_{2}$ atmosphere in Dulbecco's modified Eagle's medium (American Type Culture Collection) containing $100 \mathrm{U} / \mathrm{ml}$ penicillin, $100 \mu \mathrm{g} / \mathrm{ml}$ streptomycin (Thermo Fisher Scientific, Waltham, MA), and 10\% Hyclone fetal bovine serum (Thermo Fisher Scientific). HEK293 cells were plated at 200,000 and 800,000 cells per well in 24- or six-well plates coated with $0.1 \mathrm{mg} / \mathrm{ml}$ poly-D-lysine. Twenty-four hours later the cells were transfected following the Fugene HD protocol at the recommended 3:1 reagent to DNA ratio. For example, 24-well plates were transfected with $0.5 \mu \mathrm{g}$ plasmid DNA and $1.5 \mu \mathrm{l}$ Fugene HD (Promega, Madison, WI) per well, and uptake assays were performed 48 hours later. Medium was changed when needed.

Cell-Based Transport Assays. Cells were washed three times with prewarmed $\left(37^{\circ} \mathrm{C}\right)$ uptake buffer $\left(142 \mathrm{mM} \mathrm{NaCl}, 5 \mathrm{mM} \mathrm{KCl}, 1 \mathrm{mM} \mathrm{KH}_{2} \mathrm{PO}_{4}, 1.2 \mathrm{mM} \mathrm{MgSO}_{4}\right.$, $5 \mathrm{mM}$ glucose, and $12.5 \mathrm{mM}$ HEPES, $\mathrm{pH}$ adjusted to 7.4 with Tris base). Then, $200 \mu \mathrm{l}$ uptake buffer $\left(37^{\circ} \mathrm{C}\right)$ containing radiolabeled substrates of varying concentrations was added to the well to initiate transport for the indicated time points. Transport was terminated by four washes with ice-cold uptake buffer. Cells were lysed with $300 \mu 1 \%$ Triton X-100 in PBS at room temperature for 30 minutes prior to analysis. Two hundred microliters of cell lysate was

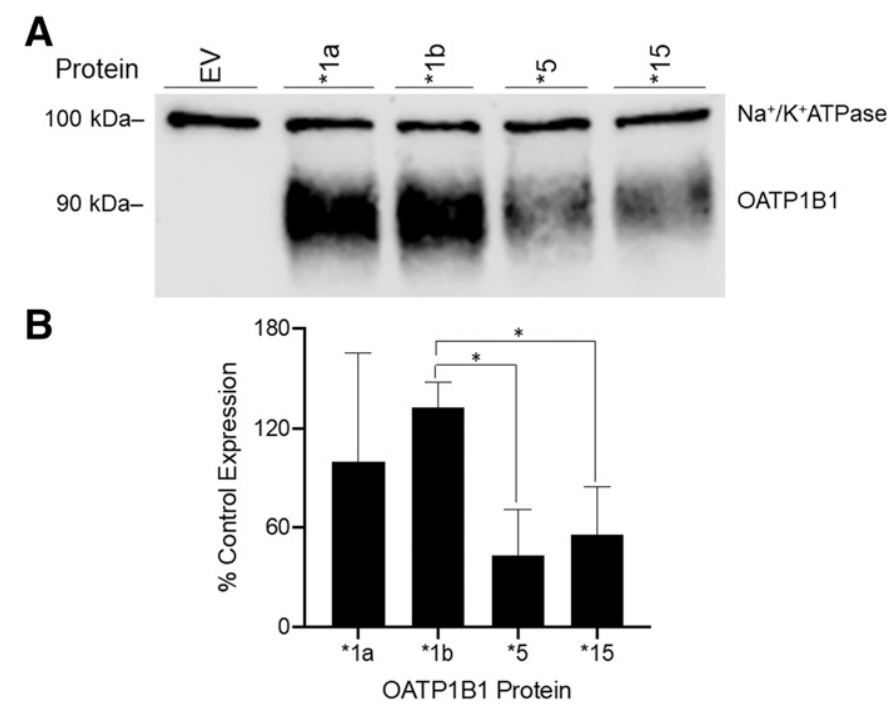

Fig. 1. Cell surface expression was evaluated via surface biotinylation experiments on HEK293 cells transfected with OATP1B1*1a, *1b, *5, and *15. Surface expressed proteins were captured with biotin, isolated used streptavidin beads, eluted, and then separated on a $7.5 \%$ polyacrylamide gel prior to being transferred to a nitrocellulose membrane. Proteins of interest were probed using a combination of $\mathrm{Na}^{+} / \mathrm{K}^{+}$-ATPase (loading control at $100 \mathrm{kDa}$ ) and tetra-His antibodies (recognizes the His-tagged OATP1B1 transporter at $\sim 86 \mathrm{kDa}$ ). (A) A representative Western blot depicting surface expression of OATP1B1 proteins is shown. (B) Western blots were then quantified using Image Studio Lite. Bars and error bars illustrate the mean surface expression \pm S.D. of the OATP1B1 proteins relative to OATP1B $1 * 1$ a from five independent experiments. Asterisks denote significant difference between OATP1B 1 proteins $(P \leq 0.05)$. 
A

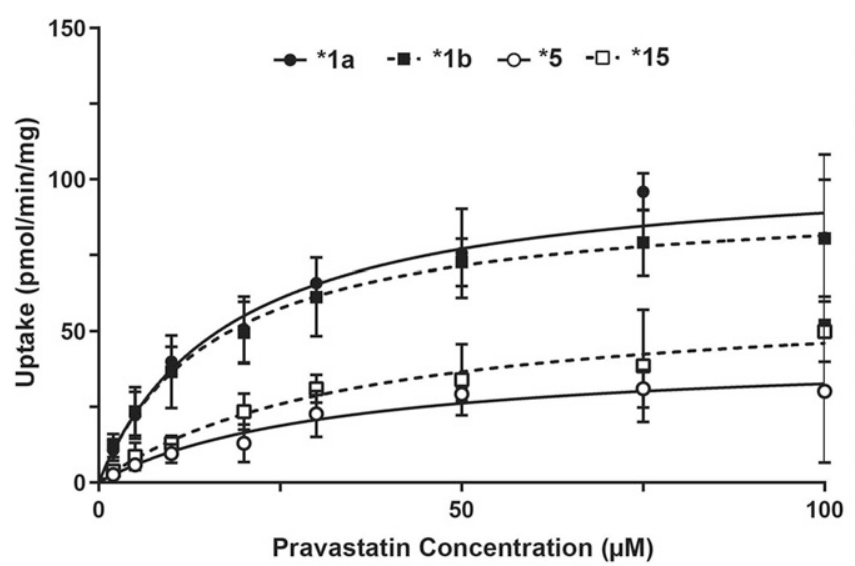

B

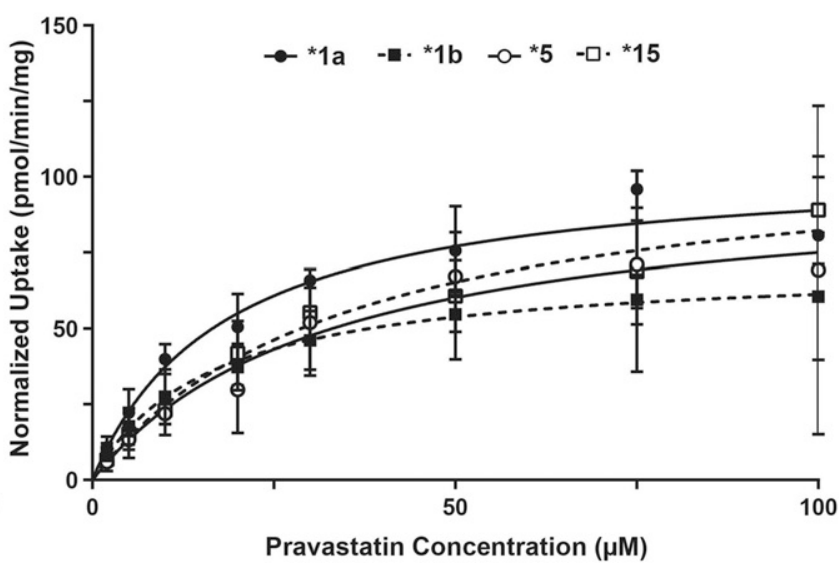

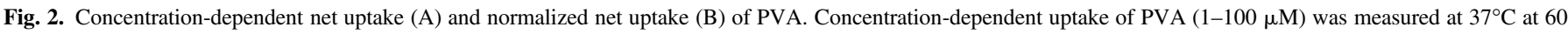

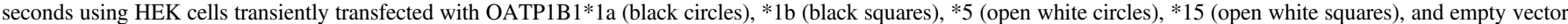

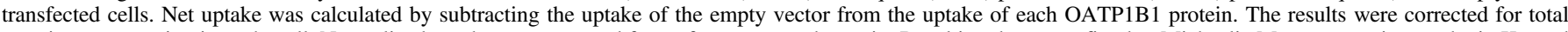

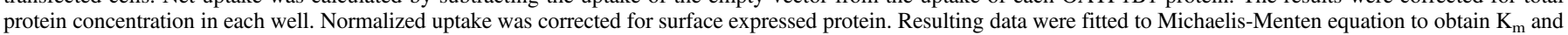
$\mathrm{V}_{\max }$ values. Each point represents the mean \pm S.D. from three to four independent experiments performed in triplicates.

transferred to a 24-well scintillation plate (Perkin Elmer, Waltham, MA), and $750 \mu l$ Optiphase Supermix scintillation cocktail (Perkin Elmer) was added to each well. Radioactivity was measured in a MicroBeta2 liquid scintillation counter (Perkin Elmer). The remaining cell lysates were transferred to 96-well plates to determine total protein concentration using the bicinchonic acid protein assay (Thermo Fisher Scientific). All transport measurements were corrected by the total protein concentration. All experiments were performed three to four times independently with two to three determinations.

Cell Surface Biotinylation. HEK293 cells were plated on six-well plates and transfected the next day as described above. Two days later, cells were first incubated on ice for 15 minutes, then washed with PBS prior to incubation with $1 \mathrm{mg} / \mathrm{ml}$ EZ-Link NHS-SS-Biotin (Thermo Fisher Scientific) in PBS at $4^{\circ} \mathrm{C}$ while rocking. After 1 hour, cells were washed with PBS to remove excess biotin and then incubated with $100 \mathrm{mM}$ glycine (Sigma Aldrich) in PBS for 20 minutes at $4^{\circ} \mathrm{C}$ while rocking. After this incubation, cells were washed again with PBS and lysed for 10 minutes on ice using lysis buffer ( $10 \mathrm{mM}$ Tris, $150 \mathrm{mM} \mathrm{NaCl}, 1 \mathrm{mM}$ EDTA, $0.1 \%$ SDS, $1 \%$ Triton $\mathrm{X}-100, \mathrm{pH} 7.5$ ) containing protease inhibitors (cOmplete protease inhibitor cocktail; Sigma Aldrich). Cell lysates were removed and centrifugated at $10,000 \mathrm{~g}$ for 2 minutes. Resulting supernatants were added to prewashed NeutrAvidin Agarose Resin beads (Thermo Fisher Scientific) for 1 hour at room temperature while rotating end-over-end. Beads were then washed with lysis buffer to remove nonbiotinylated proteins. Surface proteins were eluted from beads using $1 \times$ SDS sample buffer containing $5 \% \beta$-mercaptoethanol and $1 \times$ protease inhibitors at $70^{\circ} \mathrm{C}$ for 10 minutes and collected via centrifugation at $850 \mathrm{~g}$ for 5 minutes. Resulting samples were stored at $-80^{\circ} \mathrm{C}$ until Western blotting.

Western Blotting. To separate surface expressed proteins, samples were heated for 10 minutes at $50^{\circ} \mathrm{C}$ and then loaded onto a $7.5 \%$ polyacrylamide gel (Bio-Rad, Hercules, CA). Invitrogen's Power Blotter System was then used to transfer proteins to a nitrocellulose membrane. A blocking solution of $5 \%$ milk in Tris-buffered saline (TBS) containing $0.1 \%$ Tween 20 was used to block the blots for 1 hour at room temperature while rocking. Blots were then incubated at $4{ }^{\circ} \mathrm{C}$ overnight while rocking in blocking solution containing mouse antibodies against both the His-tag (Tetra-His Antibody, Cat. No.34670, 1:2000; QIAGEN) and the $\alpha$ subunit of $\mathrm{Na}^{+} / \mathrm{K}^{+}$-ATPase (ab7671, 1:2000; Abcam). Approximately 16 hours later, blots were washed with TBS containing $0.1 \%$ Tween 20 and then TBS before incubation for 1 hour at room temperature while rocking with goat antimouse horseradish peroxidase-conjugated secondary antibody (cat. no. 31430, 1:10,000; Thermo Fisher Scientific) in 2.5\% milk in TBS. Blots were washed with TBS and incubated for 5 minutes with SuperSignal West Pico Chemiluminescent substrate (Thermo Fisher Scientific) before visualization and quantification using Image Studio Lite Quantification Software (LI-COR, Lincoln, NE). Quantified results are from five independent experiments.

Normalization of Kinetic Parameters. The mean percentage of cell surface expression relative to the control $(* 1$ a) was used to normalize uptake values.
Normalized $\mathrm{V}_{\max }$ values for each OATP1B1 protein group were generated by dividing the mean cell surface percentage by nonnormalized $V_{\max }$ values generated from each independent experiment. These values were averaged to generate the mean \pm S.D. for each OATP1B1 protein group.

Statistical Analysis. Data were analyzed for significant differences among OATP1B1 protein groups using one-way ANOVA followed by Tukey's multiple comparison test. The familywise significance was set at 0.05 , and computed multiplicity adjusted $P$ values after Tukey's multiple comparison testing were generated using GraphPad Prism version 7 (La Jolla, CA). $\mathrm{IC}_{50}$ and kinetic parameters were calculated using nonlinear regression analysis in GraphPad Prism version 7.

\section{Results}

Cell Surface Expression of the Different OATP1B1 Proteins. When mutations are introduced into a transport protein like OATP1B1, these mutations can results in increased, decreased, or unchanged uptake. Increased or decreased uptake can be a result of more or less protein being expressed at the cell membrane. Therefore, cell membrane expression of the different OATP1B1 proteins was studied using surface biotinylation experiments. As shown in Figure 1A, there was more OATP1B1 protein expressed at the surface of HEK293 cells transfected with $\mathrm{OATP} 1 \mathrm{~B} 1 * 1 \mathrm{a}$ and $* 1 \mathrm{~b}$ than with $\mathrm{OATP} 1 \mathrm{~B} 1 * 5$ and $* 15$. This surface expression was quantitated and normalized for $\mathrm{Na}^{+} / \mathrm{K}^{+}$-ATPase, a membrane protein natively expressed in HEK293 cells. The quantification is shown in Figure 1B and Supplemental Table 1. There was not a difference in cell surface expression in OATP1B $1 * 1 \mathrm{~b}$ transfected cells compared with OATP1B $1 * 1$ a transfected cells $(133$ vs. $100, P=0.57)$.

\section{TABLE 1}

Kinetic parameters of PVA uptake by OATP1B1 proteins

All data expressed as means \pm S.D. Tukey's multiple comparisons test was used for all statistical analyses.

\begin{tabular}{lccc}
\hline OATP1B1 protein type & $\begin{array}{c}\mathrm{K}_{\mathrm{m}} \\
\mu \mathrm{M}\end{array}$ & $\begin{array}{c}\mathrm{V}_{\max } \\
\text { pmol/min per milligram }\end{array}$ & $\begin{array}{c}\mathrm{V}_{\max } / \mathrm{K}_{\mathrm{m}} \\
\mu \mathrm{l} / \mathrm{min} \text { per milligram }\end{array}$ \\
\hline$* 1 \mathrm{a}$ & $18.2 \pm 0.9$ & $104.9 \pm 13.1$ & $5.8 \pm 0.8$ \\
$* 1 \mathrm{~b}$ & $16.2 \pm 1.6$ & $94.3 \pm 16.1$ & $5.9 \pm 1.6$ \\
$* 5$ & $34.2 \pm 9.7 *, \#$ & $44.8 \pm 15.9 * *, \#$ & $1.3 \pm 0.2^{* * *, \# \#}$ \\
$* 15$ & $34.1 \pm 6.1 * \#$ & $62.3 \pm 22.5$ & $1.8 \pm 0.3^{* *, \# \#}$ \\
\hline
\end{tabular}

${ }^{*} P \leq 0.05, * * P \leq 0.01, * * * P \leq 0.001$ significantly different values from the OATP1B $1 * 1 \mathrm{a}$ ${ }^{\#} P \leq 0.05,{ }^{\# \#} P \leq 0.01,{ }^{\# \#} P \leq 0.001$ significantly different values from the OATP1B $1 * 1 \mathrm{~b}$. 
TABLE 2

Kinetic parameters of PVA uptake by OATP1B1 proteins corrected for cell surface expression

All data expressed as means \pm S.D. Tukey's multiple comparisons test was used for all statistical analyses.

\begin{tabular}{lccc}
\hline OATP1B1 protein type & $\begin{array}{c}\mathrm{K}_{\mathrm{m}} \\
\mu \mathrm{M}\end{array}$ & $\begin{array}{c}\mathrm{V}_{\max } \\
\mathrm{pmol} / \mathrm{min} \text { per milligram }\end{array}$ & $\begin{array}{c}\mathrm{V}_{\max } / \mathrm{K}_{\mathrm{m}} \\
\mu \mathrm{l} / \mathrm{min} \text { per milligram }\end{array}$ \\
\hline$* 1 \mathrm{a}$ & $18.2 \pm 0.9$ & $104.9 \pm 13.1$ & $5.8 \pm 0.8$ \\
$* 1 \mathrm{~b}$ & $16.2 \pm 1.6$ & $71.0 \pm 12.1$ & $4.5 \pm 1.2$ \\
$* 5$ & $34.2 \pm 9.7^{*}, \#$ & $103.0 \pm 36.6$ & $3.0 \pm 0.4^{* *}$ \\
$* 15$ & $34.1 \pm 6.1^{*}, \#$ & $111.2 \pm 40.2$ & $3.2 \pm 0.6^{*}$ \\
\hline
\end{tabular}

${ }^{*} P \leq 0.05, * * P \leq 0.01, * * * P \leq 0.001$ significantly different values from the OATP1B $1 * 1 \mathrm{a}$ ${ }^{\#} P \leq 0.05,{ }^{\# \#} P \leq 0.01,{ }^{\# \#} P \leq 0.001$ significantly different values from the OATP1B $1 * 1 \mathrm{~b}$.

There was no difference between OATP1B $1 * 5$ and $* 15$ transfected cells, but the two variants showed reduced expression by $57 \%$ and $44 \%$ compared with OATP1B $1 * 1 \mathrm{a}$, respectively. There was a significant decrease in OATP1B $1 * 5$ and $* 15$ expression when compared with OATP1B $1 * 1 \mathrm{~b}$ of $67 \%$ and $57 \%$, respectively. The mean percentage of cell surface expression relative to the control $(* 1$ a) was used to normalize uptake values.

PVA Uptake by HEK293 Cells Expressing the Different OATP1B1 Proteins. Initially, time-dependent uptake of PVA revealed that uptake was linear over 60 seconds (data not shown). Therefore, all concentration-dependent transport measurements for kinetic analysis were determined under initial linear rate conditions at 60 seconds. Concentration-dependent net uptake (uptake into empty vector transfected cells was subtracted from uptake by OATP1B1 transfected cells) of PVA by the different OATP1B1 variants is shown in Figure 2A. Kinetic parameters were calculated based on the Michaelis-Menten equation, and the resulting $\mathrm{K}_{\mathrm{m}}$ and $\mathrm{V}_{\max }$ values are summarized in Supplemental Table 2A and Table 1. PVA was transported by OATP1B $1 * 1 \mathrm{a}$ and $* 1 \mathrm{~b}$ transfected cells with the highest affinity ( $\mathrm{K}_{\mathrm{m}}$ values 18.2 and 16.2 , respectively) and highest maximal velocity ( $\mathrm{V}_{\max }$ values 104.9 and 94.3 , respectively) compared with OATP1B $1 * 5$ and $* 15$ transfected cells. The intrinsic clearance $\left(\mathrm{V}_{\max } / \mathrm{K}_{\mathrm{m}}\right)$ in OATP1B $1 * 1 \mathrm{a}$ and $* 1 \mathrm{~b}$ transfected cells was 3-4-fold higher compared with OATP1B1*5 and *15 transfected cells. There was no difference in the kinetic parameters between OATP1B $1 * 1 \mathrm{a}$ and $* 1 \mathrm{~b}$ transfected cells or between OATP1B $1 * 15$ and OATP1B $1 * 5$ transfected cells.
All measurements were normalized based on the cell surface expression. Corrected concentration-dependent net uptake of PVA by the different OATP1B1 variants is shown in Figure 2B. The normalized kinetic parameters with the resulting changes to maximal velocity and intrinsic clearance are summarized in Supplemental Table 2B and Table 2. The magnitude of difference in corrected intrinsic clearance between OATP1B $1 * 1$ a transfected cells and OATP1B $1 * 5$ or $* 15$ transfected cells was $\sim 2$-fold higher. However, the corrected maximal velocity was not significantly different among the four variants. There was no difference in the corrected kinetic parameters between OATP1B $1 * 1 \mathrm{a}$ and $* 1 \mathrm{~b}$ transfected cells or between OATP1B $1 * 15$ and OATP1B $1 * 5$ transfected cells.

3 $\alpha$ PVA Uptake by HEK293 Cells Expressing the Different OATP1B1 Proteins. To test whether $3 \alpha$ PVA indeed is a substrate of OATP1B1, time-dependent uptake was measured. The results (data not shown) revealed that uptake was linear over at least 60 seconds, and therefore, all concentration-dependent transport measurements for kinetic analysis were determined under these initial linear rate conditions at 60 seconds. Concentration-dependent net uptake of $3 \alpha \mathrm{PVA}$ by the different OATP1B1 variants is shown in Figure 3A. Kinetic parameters are summarized in Supplemental Table 3A and Table 3. There was no difference across all OATP1B1 proteins with respect to $3 \alpha \mathrm{PVA}$ transport affinity. $3 \alpha \mathrm{PVA}$ was transported by OATP1B1*1a and $* 1 \mathrm{~b}$ transfected cells with highest maximal velocity $\left(\mathrm{V}_{\max }\right.$ values 135.3 and 117.1 , respectively) compared with OATP1B $1 * 5$ and $* 15$ transfected cells. The intrinsic clearance in OATP1B $1 * 1$ a and $* 1 \mathrm{~b}$ transfected cells was 2 -fold higher compared with OATP1B $1 * 5$ and $* 15$ transfected cells. There was no significant difference in the kinetic parameters between OATP1B $1 * 1 \mathrm{a}$ and $* 1 \mathrm{~b}$ transfected cells or between OATP1B $1 * 15$ and OATP1B $1 * 5$ transfected cells.

After normalization for cell surface expression the results for $3 \alpha \mathrm{PVA}$ changed very similarly to the results for PVA (Fig. 3B). The corrected kinetic parameters are summarized in Supplemental Table $3 \mathrm{~B}$ and Table 4. Again, the normalized $V_{\max }$ values we not different between OATP1B $1 * 1 \mathrm{a}$ and $* 1 \mathrm{~b}$ transfected cells and $* 5$ and $* 15$ transfected cells. However, in contrast to PVA, the corrected intrinsic clearance among $\mathrm{OATP} 1 \mathrm{~B} 1 * 1 \mathrm{a}$ and $* 1 \mathrm{~b}$ transfected cells were not different compared with OATP1B1*5 and $* 15$ transfected cells. There was no difference in the corrected kinetic parameters between OATP1B $1 * 1 \mathrm{a}$ and $* 1 \mathrm{~b}$ transfected cells or between OATP1B $1 * 15$ and OATP1B $1 * 5$ transfected cells.

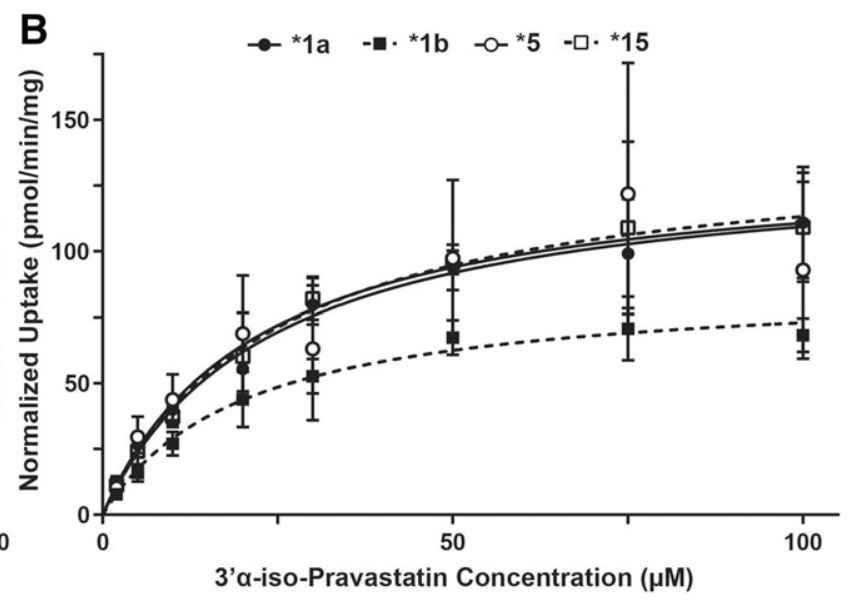

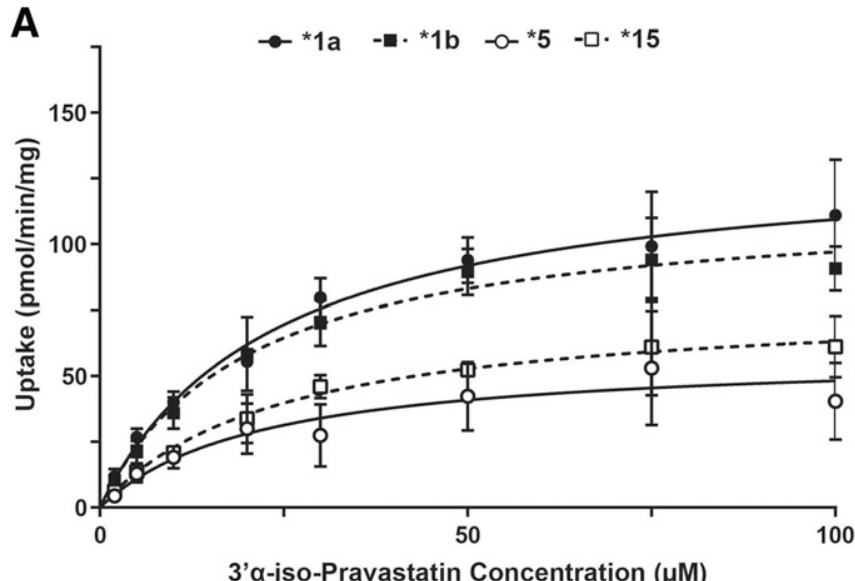

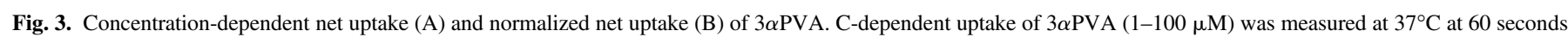

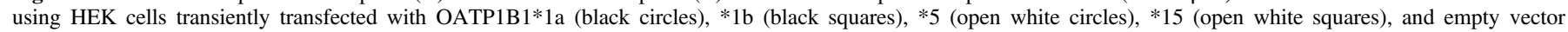

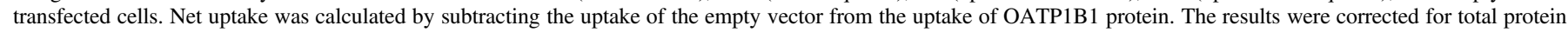

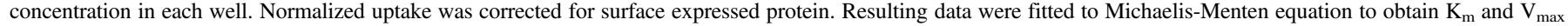
values. Each point represents the mean \pm S.D. from three to four independent experiments performed in triplicates. 
TABLE 3

Kinetic parameters of $3 \alpha$ PVA uptake by OATP1B1 proteins

All data expressed as means \pm S.D. Tukey's multiple comparisons test was used for all statistical analyses.

\begin{tabular}{lccc}
\hline OATP1B1 protein type & $\begin{array}{c}\mathrm{K}_{\mathrm{m}} \\
\mu \mathrm{M}\end{array}$ & $\begin{array}{c}\mathrm{V}_{\max } \\
\text { pmol/min per milligram }\end{array}$ & $\begin{array}{c}\mathrm{V}_{\max } / \mathrm{K}_{\mathrm{m}} \\
\mu \mathrm{l} / \mathrm{min} \text { per milligram }\end{array}$ \\
\hline$* 1 \mathrm{a}$ & $23.7 \pm 0.4$ & $135.3 \pm 5.3$ & $5.7 \pm 0.2$ \\
$* 1 \mathrm{~b}$ & $20.8 \pm 3.2$ & $117.1 \pm 8.2$ & $5.7 \pm 1.1$ \\
$* 5$ & $19.3 \pm 1.8$ & $54.2 \pm 17.7 * * * *, \# \# \#$ & $2.8 \pm 0.2^{* *, \# \#}$ \\
$* 15$ & $24.1 \pm 2.5$ & $78.3 \pm 6.8^{* * *, \# \#}$ & $3.3 \pm 0.5^{*, \#}$ \\
\hline
\end{tabular}

${ }^{*} P \leq 0.05, * * P \leq 0.01, * * * P \leq 0.001, * * * * P \leq 0.0001$ significantly different values from the OATP1B $1 * 1 \mathrm{a}$.

${ }^{\#} P \leq 0.05,{ }^{\# \#} P \leq 0.01,{ }^{\# \#} P \leq 0.001,{ }^{\# \# \#} P \leq 0.0001$ significantly different values from the $\mathrm{OATP} 1 \mathrm{~B} 1 * 1 \mathrm{~b}$

Comparison of PVA and 3aPVA Uptake among OATP1B1 Proteins. When comparing the corrected kinetic parameters for OATP1B1-mediated uptake of PVA with that of $3 \alpha \mathrm{PVA}$, there was no significant difference between the intrinsic clearances within each OATP1B1 protein with the exception of OATP1B1*5, for which $3 \alpha$ PVA intrinsic was more robust compared with PVA ( 3.0 vs. $6.4 \mu 1 / \mathrm{min}$ per milligram, $P<0.01$, Supplemental Table 4). There was no difference in $\mathrm{V}_{\max }$ values for $3 \alpha$ PVA compared with PVA within each OATP1B1 protein's cells (Supplemental Table 5). However, OATP1B1*5 had higher affinity for $3 \alpha \mathrm{PVA}$ than for PVA (19.3 vs. $34.2 \mu \mathrm{M}, P=0.01$, Supplemental Table 6). For OATP1B $1 * 15$, there was no difference between the affinity for $3 \alpha$ PVA compared with PVA (24.1 vs. $34.1, P=$ 0.17 , Supplemental Table 6).

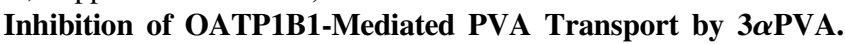
To determine what consequences the presence of $3 \alpha \mathrm{PVA}$ had on OATP1B1-mediated PVA transport, uptake of PVA was measured at low concentration $(1 \mu \mathrm{M})$ in the presence of increasing $3 \alpha \mathrm{PVA}$ concentrations. Inhibition of OATP1B1 variant-mediated uptake of PVA is shown in Figure 4. One-minute uptake into HEK293 cells transfected with $\mathrm{OATP} 1 \mathrm{~B} 1 * 1 \mathrm{a}, * 1 \mathrm{~b}, * 5, * 15$, and empty vector was measured. After uptake into empty vector transfected cells was subtracted, $\mathrm{IC}_{50}$ values were calculated by nonlinear regression analysis; the resulting values are summarized in Supplemental Table 5 and Table 5. Assuming that $3 \alpha$ PVA acts as a competitive inhibitor, $K_{i}$ values were calculated by using the previously generated PVA $K_{m}$ values for each OATP1B1 protein (Tables 1 and 2) and are summarized in Supplemental Table 7 and Table 5. The extent of inhibition of PVA transport by $3 \mathrm{aPVA}$ was similar for OATP1B $1 * 1 \mathrm{a}$ and OATP1B $1 * 1 \mathrm{~b}$; similarly, $3 \mathrm{aPVA}$ inhibited PVA uptake by OATP1B $1 * 5$ and OATP1B $1 * 15$ to a similar extent. However, $\mathrm{IC}_{50}$ and $\mathrm{K}_{\mathrm{i}}$ values were consistent with $3 \mathrm{aPVA}$ having a more marked inhibition of PVA transport by OATP1B $1 * 5$ - and *15-mediated PVA uptake compared with OATP1B1*1a- and *1bmediated uptake.

\section{Discussion}

The present study investigated whether $3 \alpha$ PVA could inhibit OATP1B1-mediated PVA uptake in an OATP1B1 variant-specific way. We found that $3 \alpha \mathrm{PVA}$ indeed inhibits PVA uptake for all OATP1B1 protein types, but most importantly, the $\mathrm{IC}_{50}$ values were significantly lower in OATP1B $1 * 5$ and $* 15$ transfected cells compared with OATP1B $1 * 1 \mathrm{a}$ and $* 1 \mathrm{~b}$ transfected cells. This suggests that a lower concentration of $3 \alpha \mathrm{PVA}$ is needed to disrupt OATP1B1-mediated PVA uptake, secondary to decreased cell surface expression of functional OATP1B1 in OATP1B $1 * 5$ and $* 15$-expressing cells. This is consistent with our previous observation that despite a similar range of PVA isomerization among genotype groups, extent of isomerization had
TABLE 4

Kinetic parameters of $3 \alpha$ PVA uptake by OATP1B1 proteins corrected for cell surface expression

All data expressed as means \pm S.D. Tukey's multiple comparisons test was used for all statistical analyses.

\begin{tabular}{lccc}
\hline OATP1B1 protein type & $\begin{array}{c}\mathrm{K}_{\mathrm{m}} \\
\mu \mathrm{M}\end{array}$ & $\begin{array}{c}\mathrm{V}_{\max } \\
\text { pmol/min per milligram }\end{array}$ & $\begin{array}{c}\mathrm{V}_{\max } / \mathrm{K}_{\mathrm{m}} \\
\mu \mathrm{l} / \mathrm{min} \text { per milligram }\end{array}$ \\
\hline$* 1 \mathrm{a}$ & $23.7 \pm 0.4$ & $135.3 \pm 5.3$ & $5.7 \pm 0.2$ \\
$* 1 \mathrm{~b}$ & $20.8 \pm 3.2$ & $88.2 \pm 6.2$ & $4.3 \pm 0.8$ \\
$* 5$ & $19.3 \pm 1.8$ & $124.6 \pm 40.8$ & $6.4 \pm 1.9$ \\
$* 15$ & $24.1 \pm 2.5$ & $140.4 \pm 11.4$ & $5.9 \pm 0.8$ \\
\hline
\end{tabular}

${ }^{*} P \leq 0.05, * * P \leq 0.01, * * * P \leq 0.001, * * * * P \leq 0.0001$ significantly different values from the OATP1B $1 * 1 \mathrm{a}$.

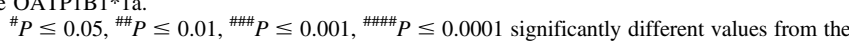
OATP1B $1 * 1 \mathrm{~b}$

a more pronounced effect on PVA systemic exposure in participants with the SLCO1B1 c.521TC variation (e.g., *5 and $* 15$ haplotypes) (Wagner et al., 2019).

There exists a dearth of human in vivo data related to variantdependent OATP1B1 expression, despite numerous studies demonstrating the impact of SLCO1B1 variant-dependent impact on OATP1B1 transporter activity (e.g., pravastatin hepatic uptake) (Mwinyi et al., 2004; Niemi et al., 2006; Wagner et al., 2019). Available proteomic data from human pediatric and adult livers demonstrate a trend toward diminished OATP1B 1 protein expression in those with $S L C O 1 B 1 * 1 a / * 15$ genotype compared with $S L C O 1 B 1$ $* 1 a / * 1 a$ genotype; however, both studies did not meet statistical significance (Prasad et al., 2014, 2016). Importantly, these studies did not report any participants with $S L C O 1 B 1 * 5$ genotype. Therefore, further proteomic investigation in larger cohorts are necessary to determine the variant-dependent OATP1B 1 expression among the full range of SLCO1B1 genotypes (e.g., *1a, *1b, *5, *15). Overall, the previous clinical observation (Wagner et al., 2019) and in vitro inhibition mechanistic observations could be a function of 1) altered OATP1B1 expression, 2) intrinsic activity, or 3) a combination of both. This variant-dependent impact on drug or metabolite inhibition with respect to transporter activity and potential for toxicity (e.g., drug-drug interaction) and/or drug response is lacking but certainly warrants more mechanistic understanding for all drug classes in the future.

In the previous pharmacogenomic-stratified pharmacokinetic studies, $3 \alpha$ PVA systemic exposure had a stronger correlation to simvastatin acid systemic exposure than PVA in a similar patient cohort (Wagner et al., 2018, 2019). This might be due to 1) $3 \alpha$ PVA being a better substrate for OATP1B1 and/or 2) $3 \alpha$ PVA transport being less affected by concurrent PVA in the system. This is in contrast to PVA transport, in which transport is disrupted by concurrent $3 \alpha \mathrm{PVA}$ in the system. Our previous SLCO1B1 pharmacogenomic-stratified pharmacokinetic investigation in participants dosed with pravastatin demonstrated a significant increase in $3 \alpha$ PVA systemic exposure in individuals with SLCO1B1 c.521T $>\mathrm{C}$ allelic variation, suggesting that it was a substrate of OATP1B1 (Wagner et al., 2019). Transcellular transport of $3 \alpha$ PVA was previously demonstrated using Madin-Darby canine kidney cells expressing OATP1B1 and multidrug resistance protein 2, suggesting that $3 \alpha \mathrm{PVA}$ is a substrate of multidrug resistance protein 2 and might be a substrate of OATP1B1 (Maeda et al., 2006). Here we confirm unequivocally that $3 \alpha \mathrm{PVA}$ is a substrate of OATP1B1 and mechanistically demonstrate that OATP1B 1 protein variation affects $3 \alpha \mathrm{PVA}$ uptake (Fig. 3). After normalization for cell surface expression, there was no significant difference in corrected intrinsic clearance for $3 \alpha \mathrm{PVA}$ among OATP1B1 proteins. This suggests that differences in $3 \alpha \mathrm{PVA}$ transport among OATP1B1 proteins are due to decreased protein 


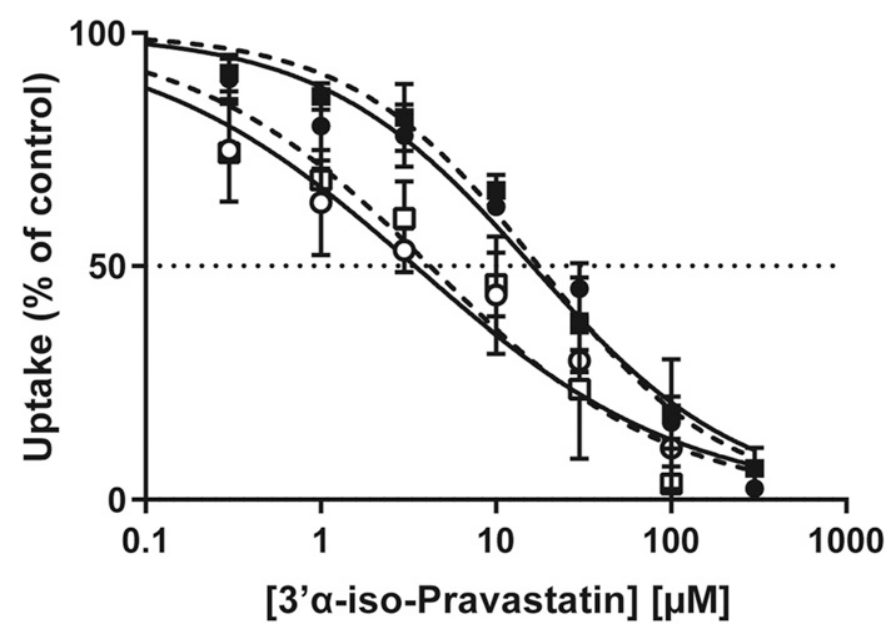

Fig. 4. Inhibition of PVA transport with $3 \alpha \mathrm{PVA}$. Uptake of low $(1 \mu \mathrm{M})$ PVA concentrations was measured at $37^{\circ} \mathrm{C}$ and 60 seconds in the presence of increasing $3 \alpha$ PVA concentrations using HEK cells transiently transfected with OATP1B $1 * 1 \mathrm{a}$ (black circles), *1b (black squares), *5 (open white circles), *15 (open white squares), and empty vector transfected cells. Net uptake was calculated by subtracting the uptake for empty vector from the uptake of the each OATP1B1 protein. The results were corrected for total protein concentration in each well. Data were reported as uptake of PVA as a percentage of control.

expression levels at the plasma membrane and not due to differences in binding affinities or turnover numbers.

$3 \alpha$ PVA was transported by all tested variants very similarly to PVA with one important exception: $\mathrm{K}_{\mathrm{m}}$ values for PVA uptake increased 2fold when OATP1B $1 * 1 \mathrm{a}$ and $* 1 \mathrm{~b}$ were compared with OATP1B $1 * 5$ and $* 15$. However, affinities for $3 \alpha \mathrm{PVA}$ were the same for all four OATP1B1 variants. As a consequence, one would expect that in patients with the $S L C O 1 B 1 * 5$ and $* 15$ genotype, $3 \alpha \mathrm{PVA}$ would preferentially be transported compared with PVA. Enhanced affinity for $3 \alpha \mathrm{PVA}$ compared with PVA in conjunction with the aforementioned inhibition would have an even more pronounced effect in subjects with the SLCO1B1 $* 5$ and $* 15$ genotypes. This could lead to a larger increase in PVA systemic exposure as compared with the other two genotypes.

This study confirmed OATP1B1-mediated PVA uptake in four OATP1B1 protein types and tested whether $3 \alpha$ PVA was indeed a substrate of OATP1B1. Our data are concordant with a previous in vitro investigation in which PVA intrinsic clearance was decreased in cells transfected with OATP1B1*5 and *15 compared with OATP1B $1 * 1 \mathrm{a}$ and $* 1 \mathrm{~b}$ (Kameyama et al., 2005). The difference in intrinsic clearances was eliminated after normalization of the uptake measurements based on cell surface expression, suggesting that the functional differences in transport observed in vivo are mainly due to alterations in protein expression. Additionally, we observed significantly higher affinity by all OATP1B1 proteins than the previous report (Kameyama et al., 2005). This could be due to slightly different experimental conditions, like shorter uptake times. Collectively, these data are consistent with previous in vivo data in adults and children in which subjects with the c.521T $>\mathrm{C}$ allelic variation had a greater risk of increased PVA systemic exposure secondary to decreased hepatocellular uptake (Mwinyi et al., 2004; Niemi et al., 2004; Wagner et al., 2019).

The clinical consequences of enhanced isomerization of PVA to $3 \alpha$ PVA are diminished systemic exposure and subsequent reduced delivery of PVA to hepatocytes (e.g., drug target) due to competition between PVA and 3 $\alpha$ PVA for OATP1B1-mediated hepatic uptake. Enhanced PVA isomerization has previously been associated with an attenuated PVA response (e.g., cholesterol reduction) (Ito, 1998). In the aforementioned study, there was no difference in $3 \alpha$ PVA systemic exposure among those subjects with excessive and diminished
TABLE 5

$3 \alpha$ PVA Inhibition of PVA uptake by OATP1B1 proteins

All data expressed as means \pm S.D. Tukey's multiple comparisons test was used for all statistical analyses. $\mathrm{K}_{\mathrm{i}}$ calculated from the equation $\mathrm{K}_{\mathrm{i}}=\mathrm{IC}_{50} /\left[1+\left([\mathrm{PVA}\right.\right.$ concentration $\left.\left.] / \mathrm{K}_{\mathrm{m}}\right)\right]$.

\begin{tabular}{lcc}
\hline OATP1B1 protein type & $\mathrm{IC}_{50}$ & $\mathrm{~K}_{\mathrm{i}}$ \\
\hline$* 1 \mathrm{a}$ & $15.9 \pm 1.9$ & $15.0 \pm 1.8$ \\
$* 1 \mathrm{~b}$ & $18.6 \pm 5.7$ & $17.5 \pm 5.4$ \\
$* 5$ & $3.9 \pm 2.0^{*, \#}$ & $3.8 \pm 2.9^{*, \#}$ \\
$* 15$ & $4.4 \pm 0.8^{*, \#}$ & $4.3 \pm 0.8^{*, \#}$ \\
\hline
\end{tabular}

${ }^{*} P \leq 0.01$ significantly different values from the OATP $1 \mathrm{~B} 1 * 1 \mathrm{a}$.

${ }^{\#} P \leq 0.01$ significantly different values from the OATP1B1*1b.

isomerization, which was defined as the ratio of PVA to $3 \alpha \mathrm{PVA}$ systemic exposure (Ito, 1998). Notably, this represents only $3 \alpha$ PVA in the plasma and not hepatic $3 \alpha \mathrm{PVA}$ concentrations. Collectively, it remains unclear whether attenuated response is secondary to 1) excessive $3 \alpha \mathrm{PVA}$ production in low $\mathrm{pH}$ environments (e.g., stomach) leaving far less active drug available systemically and/or 2) the competitive process for hepatic uptake, which is further exacerbated in SLCO1B1 c.521T $>\mathrm{C}$ variants, as mechanistically demonstrated in this study. The SLCO1B1 genotype status of both extensive and poor "gastric" metabolizer from the aforementioned study is unknown and therefore requires replication in a SLCO1B1 genotype-stratified manner.

Additionally, variable PVA isomerization not only could increase the risk of altered response (e.g., inhibition of cholesterol biosynthesis) but also could disrupt the downstream pleiotropic effects of PVA. For example, in a cohort of pediatric patients after cardiac transplantation dosed with PVA, freedom from CAV trended higher compared with those patients who did not receive statin therapy but did not reach statistical significance (Mahle et al., 2005). However, it remains unknown if the magnitude of the effect (e.g., freedom from CAV) with PVA treatment would be more significant if PVA isomerization were controlled. It remains unknown if a change in PVA formulation (e.g., enteric coating, coadministration with gastric acid buffer) would result in diminished PVA isomerization and thereby improve PVA efficacy (e.g., prevention of CAV, cholesterol reduction). Coadministration of PVA with a proton pump inhibitor or $\mathrm{H} 2$ blocker has not been previously reported but warrants future investigation. With these changes to mitigate PVA isomerization, PVA could potentially be a better agent for cholesterol reduction and other conditions of inflammatory disease.

However, there remains a knowledge gap concerning the additional dietary and physiologic factors that could be influencing PVA isomerization. For example, coadministration of PVA with food decreases the systemic exposure by $30 \%$ (Pan et al., 1993). Although food increases buffering capacity of stomach contents, food stimulates the secretion of hydrochloric acid and certainly could be influencing the diminished systemic exposure of the parent drug. In the aforementioned study by Pan et al. (1993), there was a trend toward higher systemic exposure of $3 \alpha$ PVA, but it did not meet statistical significance. Similar to the referenced study by Ito (1998), this represents only $3 \alpha$ PVA in the plasma and not hepatic $3 \alpha \mathrm{PVA}$ concentrations. Additionally, it is unknown whether reverse reaction (e.g., $3 \alpha$ PVA $\rightarrow$ PVA) could occur after absorption in the more alkaline environment of systemic circulation. Overall, the effects of PVA isomerization need to be mechanistically evaluated with altered gastric $\mathrm{pH}$ to determine if more basic $\mathrm{pH}$ results in improved intrahepatic PVA exposure and/or conversion back to PVA. It is imperative that we evaluate this, as PVA holds the lowest risk of toxicity (e.g., statin associated muscle symptoms) (Gadbut et al., 1995; Masters et al., 1995; Pierno et al., 1995; Bruckert et al., 2005) and is the safest option for the maturing child (Wagner and Abdel-Rahman, 2016). 
In conclusion, we demonstrated that $3 \alpha \mathrm{PVA}$ is an inhibitor of OATP1B1-mediated PVA uptake and has enhanced binding affinity for OATP1B1 compared with PVA, presenting another variable that could possibly result in altered PVA exposure and response for patients prescribed PVA. Moving forward, the in vitro data generated in this study can help to refine previous physiologically based pharmacokinetic models that are used to characterize PVA interindividual variability with an ultimate goal of maximizing efficacy at the lowest possible risk for toxicity.

\section{Authorship Contributions}

Participated in research design, wrote or contributed to the writing of the manuscript, performed data analysis: Wagner.

Participated in research design, performed data analysis, wrote or contributed to the writing of the manuscript: Ruggiero.

Participated in research design, wrote or conributed to the writing of the manuscript: Leeder.

Participated in research design, performed data analysis, wrote or contributed to the writing of the manuscript: Hagenbuch.

\section{References}

Bruckert E, Hayem G, Dejager S, Yau C, and Bégaud B (2005) Mild to moderate muscular symptoms with high-dosage statin therapy in hyperlipidemic patients--the PRIMO study. Cardiovasc Drugs Ther 19:403-414.

Dai R, Feng J, Wang Y, Yang Y, Deng C, Tang X, Zhao Y, Zhou H, and Zhang F (2015) Association between SLCO1B1 $521 \mathrm{~T}>\mathrm{C}$ and $388 \mathrm{~A}>\mathrm{G}$ polymorphisms and statins effectiveness: a meta-analysis. $J$ Atheroscler Thromb 22:796-815.

Everett DW, Chando TJ, Didonato GC, Singhvi SM, Pan HY, and Weinstein SH (1991) Biotransformation of pravastatin sodium in humans. Drug Metab Dispos 19:740-748.

Gadbut AP, Caruso AP, and Galper JB (1995) Differential sensitivity of C2-C12 striated muscle cells to lovastatin and pravastatin. J Mol Cell Cardiol 27:2397-2402.

Gui C and Hagenbuch B (2008) Amino acid residues in transmembrane domain 10 of organic anion transporting polypeptide 1B3 are critical for cholecystokinin octapeptide transport. Biochemistry 47:9090-9097.

Hsiang B, Zhu Y, Wang Z, Wu Y, Sasseville V, Yang WP, and Kirchgessner TG (1999) A nove human hepatic organic anion transporting polypeptide (OATP2). Identification of a liver-specific human organic anion transporting polypeptide and identification of rat and human hydroxymethylglutaryl-CoA reductase inhibitor transporters. J Biol Chem 274:37161-37168.

Igel M, Arnold KA, Niemi M, Hofmann U, Schwab M, Lütjohann D, von Bergmann K, Eichelbaum M, and Kivistö KT (2006) Impact of the SLCO1B1 polymorphism on the pharmacokinetics and lipid-lowering efficacy of multiple-dose pravastatin. Clin Pharmacol Ther 79 419-426.

Ito MK (1998) Effects of extensive and poor gastrointestinal metabolism on the pharmacodynamics of pravastatin. J Clin Pharmacol 38:331-336.

Kameyama Y, Yamashita K, Kobayashi K, Hosokawa M, and Chiba K (2005) Functional characterization of SLCO1B1 (OATP-C) variants, SLCO1B1*5, SLCO1B1*15 and SLCO1B1*15+C1007G, by using transient expression systems of HeLa and HEK293 cells. Pharmacogenet Genomics 15.513-522.

Li JH, Suchindran S, Shah SH, Kraus WE, Ginsburg GS, and Voora D (2015) SLCO1B1 genetic variants, long-term low-density lipoprotein cholesterol levels and clinical events in patients following cardiac catheterization. Pharmacogenomics 16:449-458.

Link E, Parish S, Armitage J, Bowman L, Heath S, Matsuda F, Gut I, Lathrop M, and Collins R; SEARCH Collaborative Group (2008) SLCO1B1 variants and statin-induced myopathya genomewide study. $N$ Engl J Med 359:789-799.

Luirink IK, Wiegman A, Kusters DM, Hof MH, Groothoff JW, de Groot E, Kastelein JJP, and Hutten BA (2019) 20-Year follow-up of statins in children with familial hypercholesterolemia. $N$ Engl J Med 381:1547-1556.

Maeda K, Ieiri I, Yasuda K, Fujino A, Fujiwara H, Otsubo K, Hirano M, Watanabe T, Kitamura Y, Kusuhara H, et al. (2006) Effects of organic anion transporting polypeptide 1B1 haplotype on pharmacokinetics of pravastatin, valsartan, and temocapril. Clin Pharmacol Ther 79:427-439.

Mahle WT, Vincent RN, Berg AM, and Kanter KR (2005) Pravastatin therapy is associated with reduction in coronary allograft vasculopathy in pediatric heart transplantation. J Heart Lung Transplant 24:63-66.

Martin NG, Li KW, Murray H, Putt W, Packard CJ, and Humphries SE (2012) The effects of a single nucleotide polymorphism in SLCO1B1 on the pharmacodynamics of pravastatin. $\mathrm{Br}$ $J$ Clin Pharmacol 73:303-306.
Masters BA, Palmoski MJ, Flint OP, Gregg RE, Wang-Iverson D, and Durham SK (1995) In vitro myotoxicity of the 3-hydroxy-3-methylglutaryl coenzyme A reductase inhibitors, pravastatin, lovastatin, and simvastatin, using neonatal rat skeletal myocytes. Toxicol Appl Pharmacol 131 $163-174$.

Mwinyi J, Johne A, Bauer S, Roots I, and Gerloff T (2004) Evidence for inverse effects of OATP-C (SLC21A6) 5 and 1b haplotypes on pravastatin kinetics. Clin Pharmacol Ther 75:415-421.

Nakai D, Nakagomi R, Furuta Y, Tokui T, Abe T, Ikeda T, and Nishimura K (2001) Human liverspecific organic anion transporter, LST-1, mediates uptake of pravastatin by human hepatocytes. J Pharmacol Exp Ther 297:861-867.

Niemi M, Neuvonen PJ, Hofmann U, Backman JT, Schwab M, Lütjohann D, von Bergmann K, Eichelbaum M, and Kivistö KT (2005) Acute effects of pravastatin on cholesterol synthesis are associated with SLCO1B1 (encoding OATP1B1) haplotype *17. Pharmacogenet Genomics 15: 303-309.

Niemi M, Pasanen MK, and Neuvonen PJ (2006) SLCO1B1 polymorphism and sex affect the pharmacokinetics of pravastatin but not fluvastatin. Clin Pharmacol Ther 80:356-366.

Niemi M, Schaeffeler E, Lang T, Fromm MF, Neuvonen M, Kyrklund C, Backman JT, Kerb R, Schwab M, Neuvonen PJ, et al. (2004) High plasma pravastatin concentrations are associated with single nucleotide polymorphisms and haplotypes of organic anion transporting polypeptideC (OATP-C, SLCO1B1). Pharmacogenetics 14:429-440.

Pan HY (1991) Clinical pharmacology of pravastatin, a selective inhibitor of HMG-CoA reductase. Eur J Clin Pharmacol 40 (Suppl 1):S15-S18.

Pan HY, DeVault AR, Brescia D, Willard DA, McGovern ME, Whigan DB, and Ivashkiv E (1993) Effect of food on pravastatin pharmacokinetics and pharmacodynamics. Int J Clin Pharmacol Ther Toxicol 31:291-294.

Pasanen MK, Backman JT, Neuvonen PJ, and Niemi M (2006) Frequencies of single nucleotide polymorphisms and haplotypes of organic anion transporting polypeptide 1B1 SLCO1B1 gene in a Finnish population. Eur J Clin Pharmacol 62:409-415.

Pierno S, De Luca A, Tricarico D, Roselli A, Natuzzi F, Ferrannini E, Laico M, and Camerino DC (1995) Potential risk of myopathy by HMG-CoA reductase inhibitors: a comparison of pravastatin and simvastatin effects on membrane electrical properties of rat skeletal muscle fibers. J Pharmacol Exp Ther 275:1490-1496.

Prasad B, Evers R, Gupta A, Hop CE, Salphati L, Shukla S, Ambudkar SV, and Unadkat JD (2014) Interindividual variability in hepatic organic anion-transporting polypeptides and P-glycoprotein (ABCB1) protein expression: quantification by liquid chromatography tandem mass spectroscopy and influence of genotype, age, and sex. Drug Metab Dispos 42:78-88.

Prasad B, Gaedigk A, Vrana M, Gaedigk R, Leeder JS, Salphati L, Chu X, Xiao G, Hop C, Evers R, et al. (2016) Ontogeny of hepatic drug transporters as quantified by LC-MS/MS proteomics. Clin Pharmacol Ther 100:362-370.

Serajuddin AT, Ranadive SA, and Mahoney EM (1991) Relative lipophilicities, solubilities, and structure-pharmacological considerations of 3-hydroxy-3-methylglutaryl-coenzyme A (HMGCoA) reductase inhibitors pravastatin, lovastatin, mevastatin, and simvastatin. J Pharm Sci 80 830-834.

Shitara Y and Sugiyama Y (2006) Pharmacokinetic and pharmacodynamic alterations of 3-hydroxy-3-methylglutaryl coenzyme A (HMG-CoA) reductase inhibitors: drug-drug interaction and interindividual differences in transporter and metabolic enzyme functions. Pharmacol Ther 112:71-105.

Suwannakul S, Ieiri I, Kimura M, Kawabata K, Kusuhara H, Hirota T, Irie S, Sugiyama Y, and Higuchi S (2008) Pharmacokinetic interaction between pravastatin and olmesartan in relation to SLCO1B1 polymorphism. J Hum Genet 53:899-904.

Tachibana-Iimori R, Tabara Y, Kusuhara H, Kohara K, Kawamoto R, Nakura J, Tokunaga K Kondo I, Sugiyama Y, and Miki T (2004) Effect of genetic polymorphism of OATP-C (SLCO1B1) on lipid-lowering response to HMG-CoA reductase inhibitors. Drug Metab Pharmacokinet 19:375-380.

Triscari J, O'Donnell D, Zinny M, and Pan HY (1995) Gastrointestinal absorption of pravastatin in healthy subjects. J Clin Pharmacol 35:142-144.

Voora D, Shah SH, Spasojevic I, Ali S, Reed CR, Salisbury BA, and Ginsburg GS (2009) The SLCO1B $1 * 5$ genetic variant is associated with statin-induced side effects. $J$ Am Coll Cardiol 54 $1609-1616$.

Wagner J and Abdel-Rahman SM (2016) Pediatric statin administration: navigating a frontier with limited data. J Pediatr Pharmacol Ther 21:380-403.

Wagner JB, Abdel-Rahman S, Gaedigk R, Gaedigk A, Raghuveer G, Staggs VS, Kauffman R, Van Haandel L, and Leeder JS (2019) Impact of genetic variation on pravastatin systemic exposure in pediatric hypercholesterolemia. Clin Pharmacol Ther 105:1501-1512.

Wagner JB, Abdel-Rahman S, Van Haandel L, Gaedigk A, Gaedigk R, Raghuveer G, Kauffman R, and Leeder JS (2018) Impact of SLCO1B1 genotype on pediatric simvastatin acid pharmacokinetics. J Clin Pharmacol 58:823-833.

Address correspondence to: Jonathan B. Wagner, Ward Family Heart Center and Division of Clinical Pharmacology, Toxicology and Therapeutic Innovation, Children's Mercy Kansas City, 2401 Gillham Road, Kansas City, MO 64108. E-mail: jbwagner@cmh.edu 